\title{
Experimentally induced fat embolism syndrome: shift from obstruction to toxic effects
}

\author{
Hong Il Kim, Seok Kyung In, \\ Hyung Suk Yi, Hyo Young Kim, \\ Yoon Soo Kim
}

Department of Plastic and Reconstructive Surgery, Kosin University Gospel Hospital, Busan, Korea

\begin{abstract}
Background Two hypothetical mechanisms have been proposed for fat embolism syndrome: mechanical obstruction and biochemical reactions. However, it has not been proven whether these mechanisms are correlated. This study aimed to demonstrate the relationship between these two hypothetical mechanisms by observing biochemical and histological changes in animals.

Methods After a preliminary study, $700 \mathrm{mg} / \mathrm{kg}$ of triolein was injected via the ear vein into 25 rabbits and hemodynamic changes in triglycerides, lipases, free fatty acids, and albumin over time were observed. Necropsies were immediately conducted on all experimental animals, and the lungs were examined histologically.

Results Eight rabbits died within 1 hour after the injection due to mechanical obstruction. Six rabbits died 7-60 hours after the injection due to diffuse hemorrhage of the lung induced by the toxic biochemical reactions of free fatty acids. Histological examinations of the lungs of the surviving rabbits showed petechiae on the surfaces and evidence of recovery from hemorrhage. Blood levels of free fatty acids increased immediately after the injection of triolein.

Conclusions This study revealed that fat emboli primarily injure the lung via mechanical obstruction. The fat is hydrolyzed into fatty acids and causes secondary damage via biochemical reactions. The present study sheds light on the pathophysiology of fat embolism syndrome, with possible implications for its management and prevention.
\end{abstract}

Keywords Embolism / Fat / Fatty acids / Triglycerides

\section{INTRODUCTION}

Liposuction, which is widely performed in the field of plastic surgery, was recently identified as a necessary process for the harvesting of adipose-derived stem cells [1]. In liposuction, fat tissue is crushed and removed using physical force. An inevitable consequence of liposuction is damage to the small vessels, through which

Received: Sep 19, 2020 Revised: Nov 16, 2020 Accepted: Dec 3, 2020 Correspondence: Yoon Soo Kim Department of Plastic and Reconstructive Surgery, Kosin University Gospel Hospital, 262 Gamcheon-ro, Seo-gu, Busan 49267, Korea

Tel: +82-51-990-6131, Fax: +82-51-990-6312, E-mail: medissu@naver.com

Copyright @ 2021 The Korean Society for Aesthetic Plastic Surgery.

This is an Open Access article distributed under the terms of the Creative Commons Attribution Non-Commercial License (https://creativecommons.org/licenses/by-nc/4.0/) which permits unrestricted non-commercial use, distribution, and reproduction in any medium, provided the original work is properly cited. $\quad w w w . e-a a p s . o r g$ shredded pieces of fat (fat emboli) can then flow; this phenomenon is known as fat embolism. If a pathologic state results, it is called fat embolism syndrome (FES) [2]. In FES, fat emboli are not fat tissue fragments or fat cells; rather, they are lipid drops floating in the circulation [3].

FES was first reported by Zenker [4] in 1862. Both fulminantacute and sub-acute FES, which are caused by an abnormal influx of fat into the blood vessels, are associated with characteristic clinical symptoms, including petechiae, confusion, and acute respiratory failure. FES can have fatal complications. Fortunately, most instances of fat embolism are subclinical [5].

Fat, the causative agent of FES, can be divided into two types: transported fat, which is present in the blood; and stored fat, which is located outside the blood vessels. Transported fat is in the form of chylomicrons bound to apolipoprotein, whereas stored fat accumulates within adipocytes as triglycerides (TGs). TGs can be hydrolyzed to free fatty acids (FFAs) by lipase, and FFAs bound to al- 
bumin are then moved to other tissues through the blood vessels.

FFAs, a toxic substance, bind calcium ions in the blood and breaks the junctions between endothelial cells. This disrupts the endothelial cell layer surface of capillaries and increases vascular permeability, resulting in hemorrhage and edema [5]. FFAs can attach to neutrophils, which play a significant role in inflammation and increase the expression of CD11b/CD18 $\beta_{2}$ integrins on neutrophils. Neutrophils produce proteases that digest alveolar endothelial cells [6].

FES progresses rapidly, but few studies have examined its prediction, management, or prophylaxis [7]. Therefore, it is important to improve our understanding of its pathophysiology.

The progression of subclinical fat embolism to significant FES can be explained by two hypothetical mechanisms. The first theoretical mechanism is mechanical obstruction, in which peripheral vessels are mechanically obstructed by fat emboli when adipose tissue is damaged and the end organs subsequently develop ischemia and severe dysfunction, called fulminant-acute FES [8]. A significant quantity of fat emboli is needed to cause clinical symptoms, which progress very quickly. However, cases of FES caused by mechanical obstruction are very rare. The other proposed mechanism is the biochemical theory, according to which FFAs destroy organs through chemical interactions and cause sub-acute FES [9]. The more frequent type, sub-acute FES, usually occurs in 12-48 hours after an injury. Peltier [10] first described these two theories in animal experiments by injecting TGs and FFAs. However, no studies to date have examined the interaction between these two causative agents of FES.

In FES, fat emboli that enter the venous circulation are TGs (stored fat), not FFAs. Therefore, the two main theories of the etiology of FES, mechanical obstruction and biochemical mechanisms, are likely related and do not act independently. In other words, if the amount of fat entering the bloodstream is insufficient to induce fulminant-acute FES, the remaining fat will be hydrolyzed to FFAs. This results in a shift from mechanical obstruction to the biochemical phase, which leads to sub-acute FES. However, there is no evidence that fat emboli that cause mechanical obstruction are hydrolyzed to FFAs and cause FES through the toxic biochemical action of FFAs.

The present study was designed to test the hypothesis that fat emboli that mechanically obstruct alveolar capillaries are hydrolyzed to FFAs, causing sub-acute FES. To induce fat embolism, triolein, a representative stored fat, was injected to the veins of rabbits. The linear formula of triolein is $\left(\mathrm{C}_{17} \mathrm{H}_{33} \mathrm{COOCH}_{2}\right)_{2} \mathrm{CHOCOC}_{17} \mathrm{H}_{33}$; its chemical formula is $\mathrm{C}_{57} \mathrm{H}_{104} \mathrm{O}_{6}$, its molecular weight is $885.45 \mathrm{~g} /$ $\mathrm{mol}$, and its density is $0.913 \mathrm{mg} / \mathrm{mL}$. We measured changes in the concentration of some blood lipids shortly after the triolein injection. In necropsies, we examined the lung tissues histologically to investigate the relationship between changes in blood FFA levels and the development and progression of FES.

\section{METHODS}

\section{Preliminary study}

In a preliminary study, we used eight rabbits to determine the lethal dose of triolein. Doses of 200, 300, 400, 500, 600, 700, 800, or $900 \mathrm{mg} / \mathrm{kg}$ of triolein were injected into the ear vein of each rabbit via a syringe pump (TE-311; Terumo Co., Tokyo, Japan) at 300 $\mathrm{mL} / \mathrm{hr}$. The rabbits into which triolein doses of $200,300,400,500$, 600 , or $700 \mathrm{mg} / \mathrm{kg}$ were injected survived, while those into which triolein doses of 800 or $900 \mathrm{mg} / \mathrm{kg}$ were injected died within 1 hour. Accordingly, $700 \mathrm{mg} / \mathrm{kg}$ of triolein was chosen as the injection dose to avoid the development of fulminant-acute FES.

\section{Experimental animals}

Thirty-three New Zealand white rabbits (healthy 9-week-old males; mean weight, $2 \mathrm{~kg}$; range, 1.9-2.1 kg; Samtako Co., Osan, Korea) were used in the present and preliminary studies. We used triolein (triolein, non-emulsion type; Kanto Chemical Co. Inc., Tokyo, Japan), a representative form of stored fat, to induce fat embolism. Based on the preliminary study, $700 \mathrm{mg} / \mathrm{kg}$ of triolein was the injection dose used in all rabbits to avoid the development of fulminant-acute FES. The experimental and animal care procedures were approved by the Institutional Animal Care and Use Committee of Good Moonhwa Hospital (No. 2013-02) and followed the principles of scientific research ethics.

\section{Biochemical testing}

Triolein $(700 \mathrm{mg} / \mathrm{kg} ; 1.53 \mathrm{~mL})$ was injected into 25 rabbits through an ear vein by a syringe pump (TE-311, Terumo Co.) at $300 \mathrm{~mL} / \mathrm{hr}$. Blood samples were collected before the injection and at 3, 12, 24, 48 , and 72 hours after the injection. We also measured the concentrations of blood lipids, such as TGs, FFAs, and lipase, as well as albumin. To minimize changes in blood levels induced by feeding, the rabbits were fasted for at least 8 hours prior to the blood sampling.

\section{Histological examinations}

Necropsies were performed immediately after death or the end of the experiment. After gross examination of the internal organs, particularly the lungs and heart, both lungs were removed for microscopic examinations. One side of each lung was fixed in $10 \%$ formalin and embedded in paraffin. Conventional hematoxylin and eosin (H\&E) staining was performed. The other side of each lung was quick-frozen and sectioned to determine the presence and distribution of fat by Sudan Black B staining. The sections were observed under a microscope from the hilar portion of the lung, including the terminal regions of the alveoli and capillaries. 


\section{RESULTS}

Among the 25 rabbits included in the study, eight died within 1 hour after the injection, while another six died 7-60 hours after the injection. The other 11 survived to the end of the experiment.

Rabbits that died immediately after the triolein injection Each of the eight rabbits that died within 1 hour of the injection showed an enlarged right ventricle as well as pale and ischemic lungs (Fig. 1). The lung tissue stained with H\&E and Sudan Black $B$ was examined under a microscope. There was no evidence of vessel wall destruction or hemorrhage. The large vessels were filled
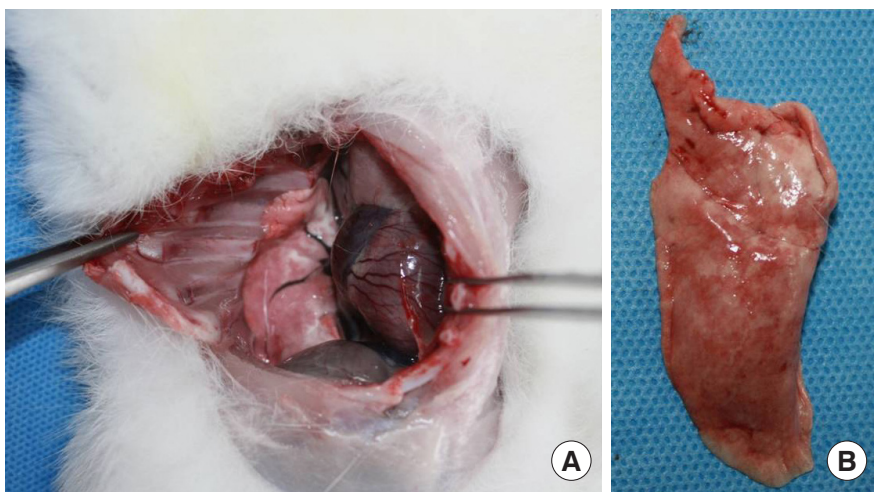

Fig. 1. Autopsy after the triolein injection. (A) Open right side of the chest of a rabbit that died immediately after the injection of triolein, showing an enlarged right heart and no hemorrhage. (B) The lung removed from a rabbit that died immediately after the injection of triolein is pale due to ischemic changes caused by the fat embolism. with large fat globules (Fig. 2). Sudan Black B staining showed black fat globules within the large vessels and some of the interalveolar vessels (Fig. 3).

Rabbits that died 7-60 hours after the triolein injection One rabbit each died at 7, 10,16,17,27, and 60 hours after the injection. Each showed slowly progressing dyspnea and unconsciousness starting 6 hours after the injection. The necropsy findings showed diffuse hemorrhaging in the lungs (Fig. 4). Cardiac hyper-

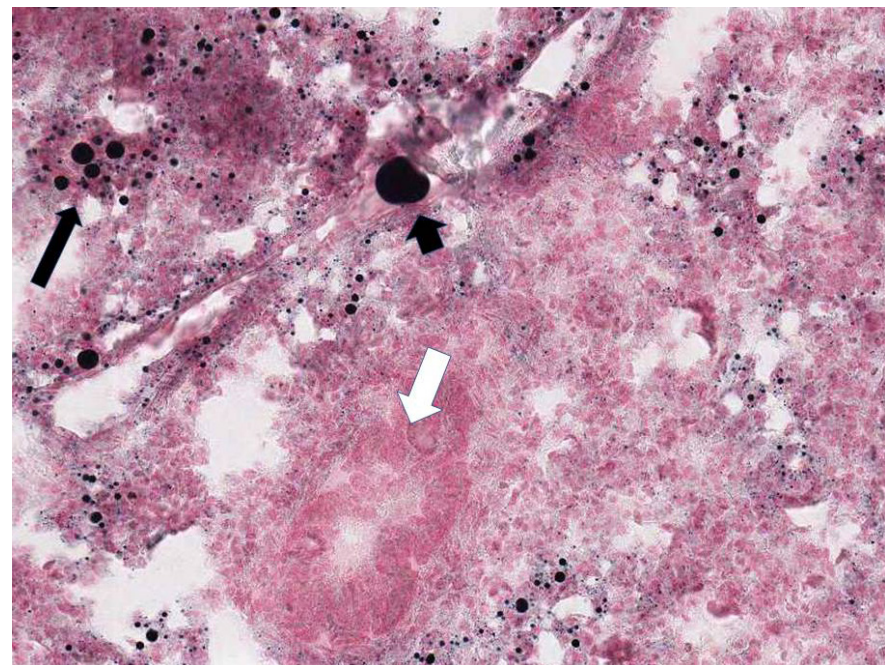

Fig. 3. A black large fat vacuole (short black arrow) and numerous small fat globules (long black arrow) are visible in the vessels of the lung, but not in the bronchial wall in the center (white arrow) (Sudan Black B stain, $\times 100$ ).
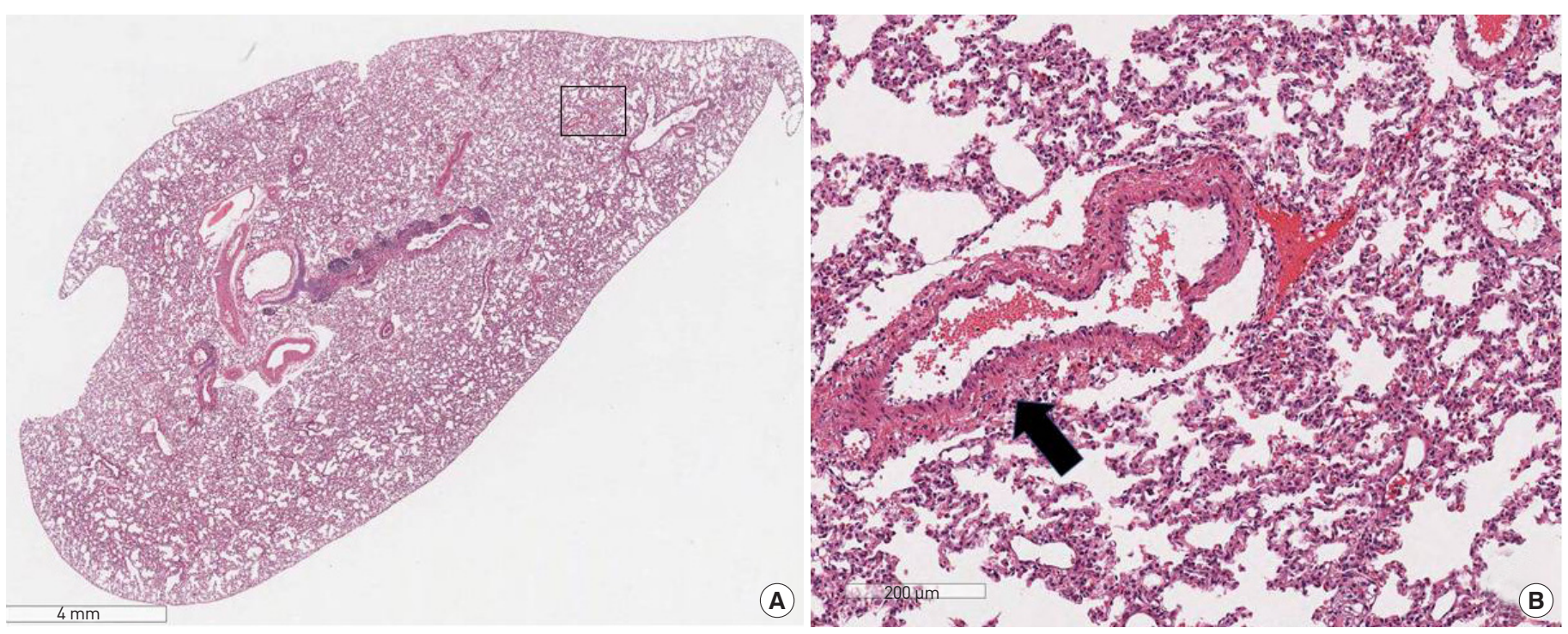

Fig. 2. Histopathologic findings. (A) Transverse section of the lung from the hilum to the periphery (H\&E, $\times 1)$. (B) High-power view of panel $A$. The black square shows aggregated large fat vacuoles packed in a thick-walled large arterial wall (black arrow), whereas no remarkable change is visible in the lung parenchyma except for capillary congestion of the interalveolar septa $(H \& E, \times 100)$. 


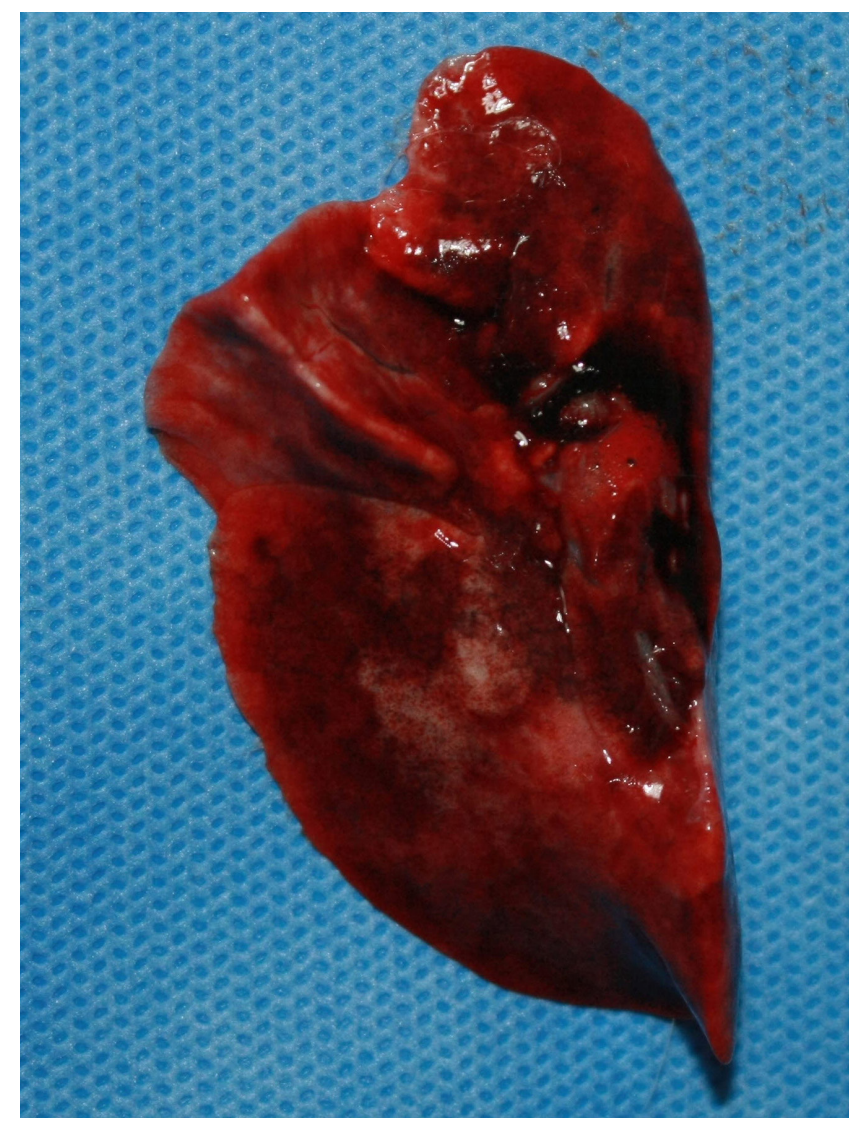

Fig. 4. Visible diffuse hemorrhage in a lung removed from the rabbit that died 10 hours after the injection of triolein. trophy was not observed. Hemorrhagic fluid was found in the pleural cavity. Microscopic examination of the lung showed diffuse hemorrhage, extravasated red blood cells, and edematous fluid in the alveoli. Many clear spaces of variable size appearing to be fat vacuoles mixed with blood were present in the medium-sized vessels. Numerous small, clear spaces of fat vacuoles were visible in the alveoli and alveolar capillaries (Fig. 5). Fat staining showed fat

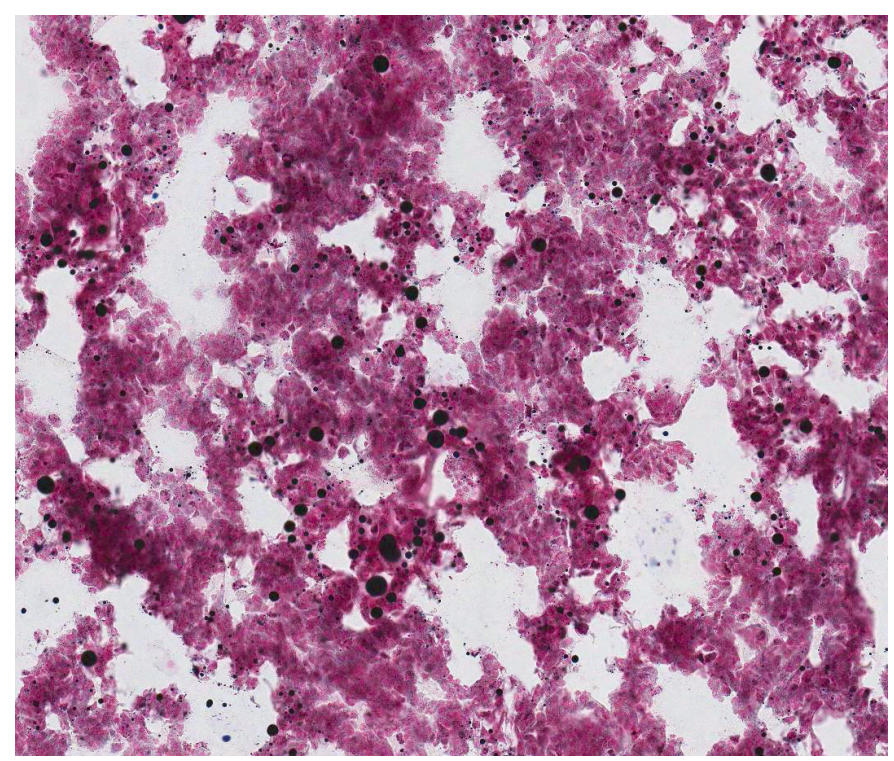

Fig. 6. Diffusely scattered, variably sized black fat globules visible in vessels and bronchial spaces of the lung (Sudan Black B stain, $\times 100$ ).
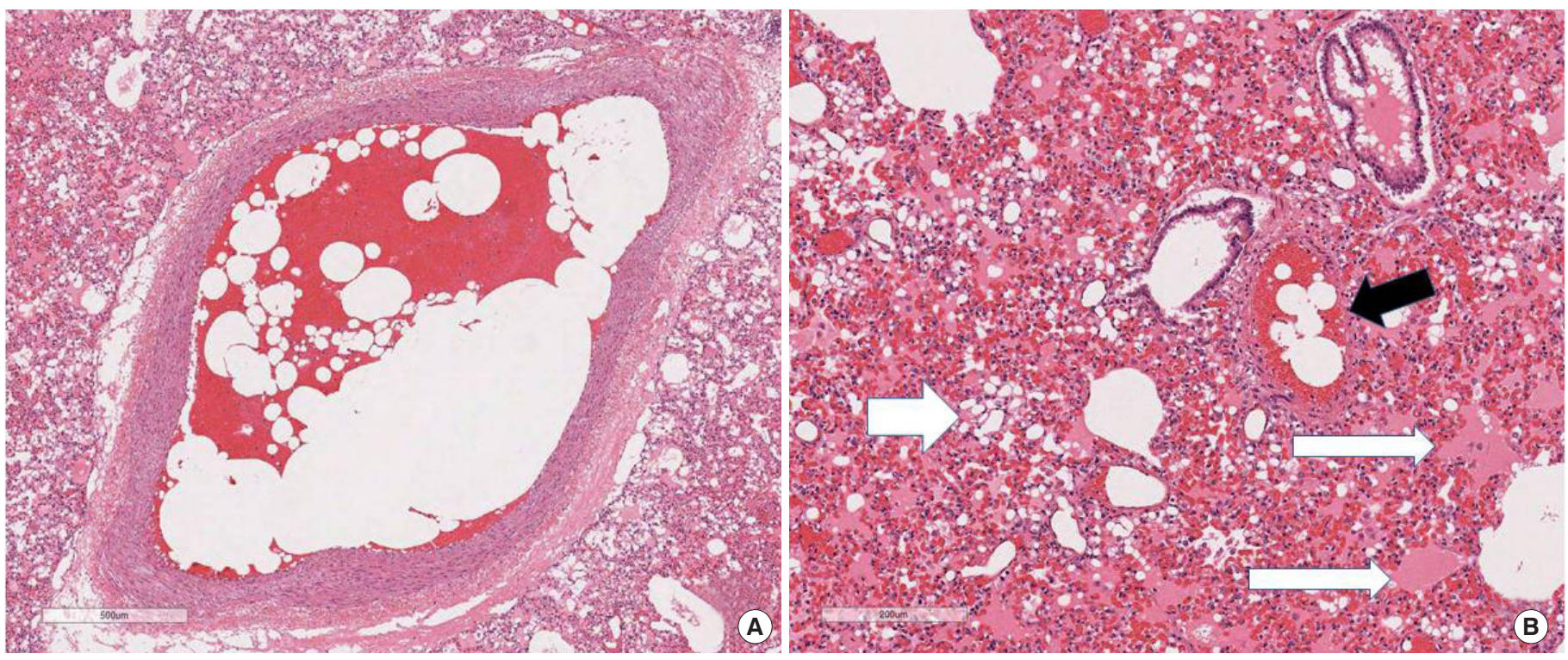

Fig. 5. Histopathologic findings. (A) Transverse section from the hilum to the periphery. The square shows large clear spaces of fat vacuoles mixed with blood within a medium-sized artery (H\&E, ×40). (B) Many variable-sized clear spaces of fat vacuoles mixed with blood within a medium-sized artery (black arrow), diffuse hemorrhaging and edematous fluid in the alveoli (white long arrows), and numerous small clear spaces of fat vacuoles within the alveoli and alveolar capillaries (white short arrow) (H\&E, $\times 100)$. 
Table 1. Changes in triglyceride, lipase, free fatty acid, and albumin levels in the 11 rabbits that survived fat embolism

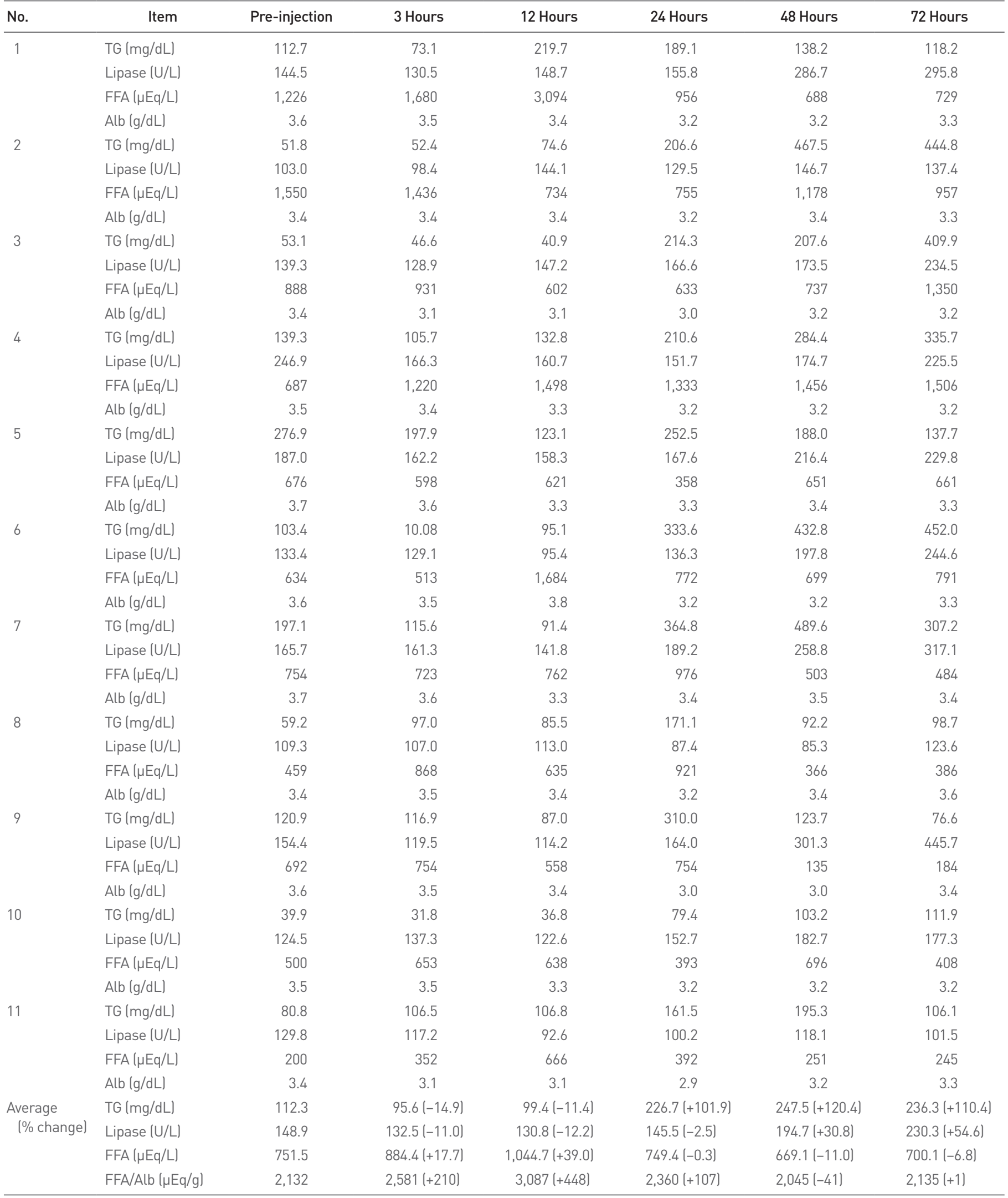

TG, triglyceride; FFA, free fatty acid; Alb, albumin. 


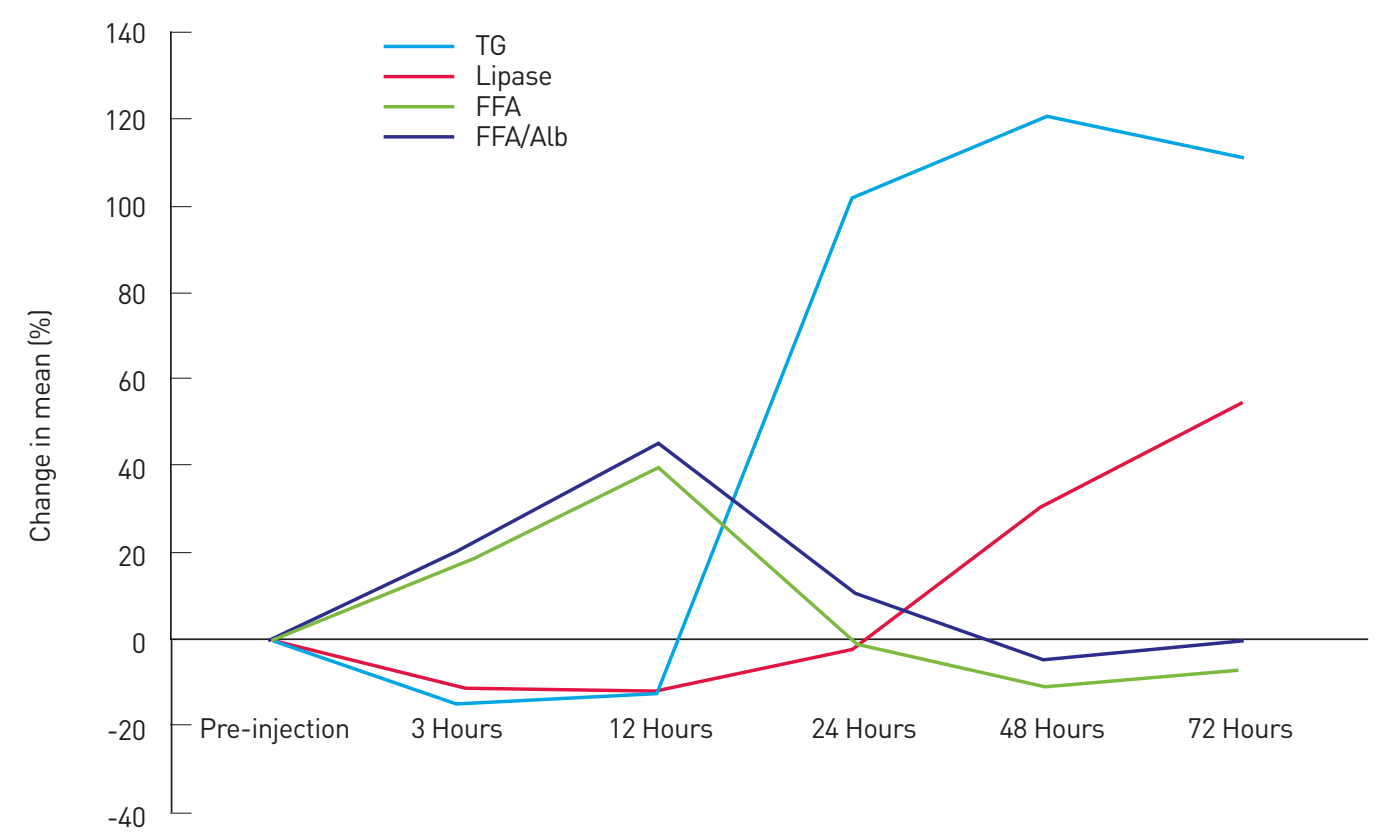

Fig. 7. Change in mean TG, lipase, FFA, and FFA/Alb levels in the 11 surviving rabbits lusing the mean pre-injection measurement as the baseline, increases and decreases are indicated as percentages). TG, triglyceride; FFA, free fatty acid; Alb, albumin.

globules diffusely scattered throughout the lung (Fig. 6). Biochemical tests showed that the FFA levels of these rabbits were markedly higher than those of the surviving rabbits (Table 1).

\section{Rabbits that survived until the end of the experiment}

Eleven of the 25 rabbits survived to the end of the experiment. The blood concentrations of TGs, FFAs, lipase, and albumin were measured over time. There were some differences among individual subjects in the changes of these four parameters. However, as the mean FFA level began to increase 3 hours after the injection, the mean TG and lipase levels slightly decreased. After 12 hours, the FFA level rapidly decreased, and the TG level began to increase markedly. The lipase level increased by 24 hours after the injection, while the TG level remained high until the end of the experiment. After 48 hours, the FFA level increased slightly and returned to the pre-injection level. To detect pure FFA, which is not bound to albumin, the FFA/albumin level was also measured. The changes of FFA/albumin did not differ from those of FFAs (Fig. 7). Tachypnea was observed in each surviving rabbit, but tended to resolve over time. Focal hemorrhagic areas and petechiae were observed in the lungs (Fig. 8). The lungs showed mixed areas of multifocal hemorrhaging, petechiae, and normal lung tissue on the cut surface (Fig. 9A). The H\&E and Sudan Black B stains showed blood vessel destruction and hemorrhagic areas admixed with normal lung tissue. In the hemorrhagic areas, many alveolar macrophages were seen phagocytosing red blood cells and filling the alveoli. Some large, clear spaces of fat vacuoles remained in the alveoli (Fig. 9B). Fat staining showed black fat globules of variable sizes scattered multi-

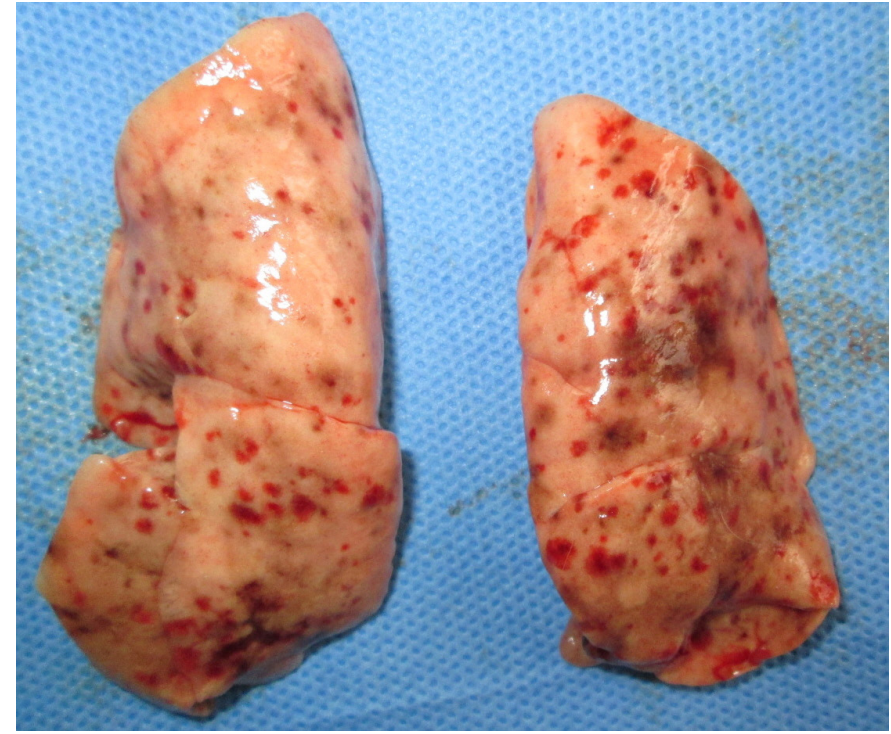

Fig. 8. Examination of the lung removed from a rabbit that survived for 72 hours after the injection of triolein, showing multifocal reddish-brown petechiae throughout.

focally in the lung (Fig. 9C).

\section{DISCUSSION}

Fat embolism is defined as the mechanical obstruction of blood vessels, especially the capillaries, due to the presence of small circulating fat droplets of $7-14 \mu \mathrm{m}$ in diameter $[3,11]$. 

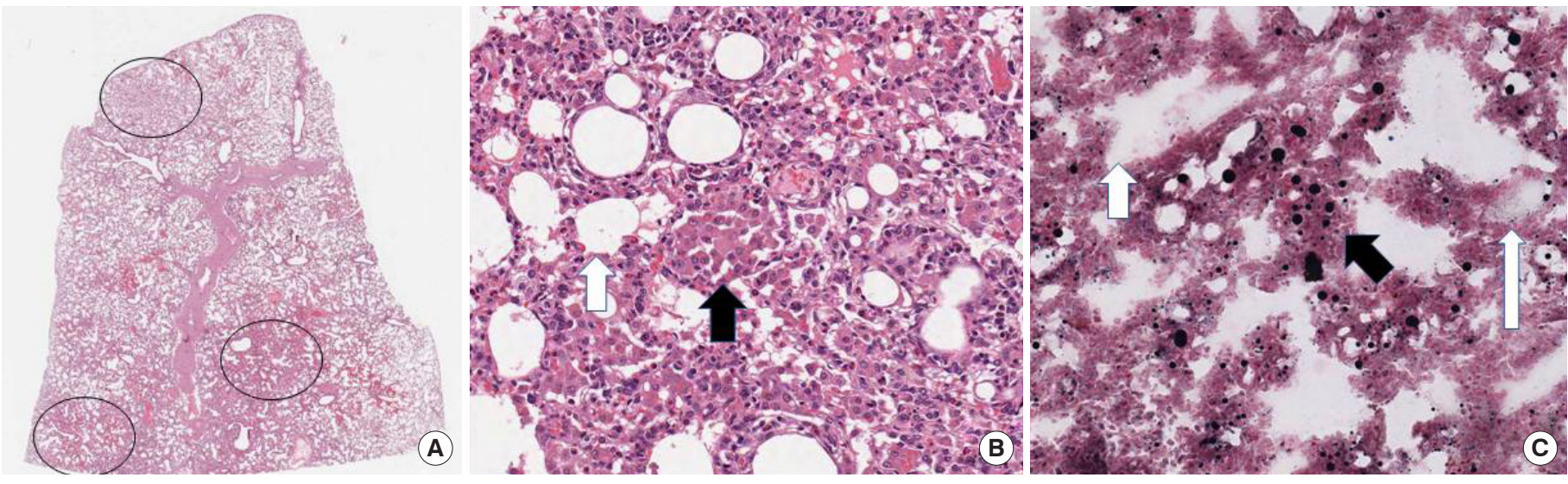

Fig. 9. Histopathologic findings. (A) The lung contains mixed areas of normal tissue and hemorrhagic lesions (black circle) (H\&E, $\times 1$ ). (B) The lung removed from a rabbit that survived 72 hours after injection of triolein contains many macrophages phagocytizing red blood cells (black arrow) and some clear spaces of fat vacuoles in the alveoli (white arrow) (H\&E, $\times 200)$. (C) Diffusely scattered black fat globules of various sizes are visible in the vessels (black arrow) of the lung, whereas some portions of the bronchial wall contain fat globules (white long arrow) and others do not (white short arrow) (Sudan black B stain, $\times 100$ ).

FES involves the functional deficit of one or more organs due to damage by fat emboli [3]. Because fat emboli initiate and expand throughout the venous circulation, the lungs are primarily involved. However, any body organ or tissue can be affected when fat emboli pass through the pulmonary capillaries or the atrial septum [12]. These findings have been described in some autopsy reports and animal studies in which fat emboli were found in the kidneys and brains after severe injury [13,14].

FES usually results from high-energy trauma in association with a long-bone or hip fracture [15]. The incidence of FES is high in orthopedic trauma patients, but it can occur in cases of septicemia, sickle cell anemia, pancreatitis, diabetes mellitus, fatty liver, longterm steroid use, and extensive burns [3,16,17]. FES also occurs frequently after cosmetic procedures such as liposuction and fat injection [11,18-22]. The fat emboli caused by fractures are derived from the bone marrow fat, while those caused by liposuction are subcutaneous fat in origin; however, both sources contain the same kind of stored fat.

The mechanism of FES is very complex, and two current hypotheses exist. The first is the mechanical obstruction theory proposed by Gauss [8] in 1924. In this theory, symptoms appear due to mechanical obstruction of the pulmonary capillaries by fat emboli. In such cases, if the fat emboli obstruct more than $80 \%$ of the pulmonary capillaries, pulmonary arterial pressure increases and falls, causing acute right ventricular failure, which rapidly leads to death $[7,12,23]$. The second is the biochemical theory introduced by Lehman and Moore [9] in 1927. According to this theory, FFAs, which are toxic, bind with calcium ions in the blood and disrupt the junctions between endothelial cells, disrupting the endothelial cell layer of capillaries, increasing vascular permeability, and resulting in diffuse hemorrhaging and edema [3]. In addition, neutro- phils play a significant role in the cell damage that occurs during inflammation $[5,6]$. The toxic action of FFAs seriously damages the alveoli and capillaries, resulting in hemorrhage.

We propose that these two hypotheses may not be independent. If the amount of fat emboli is insufficient to initiate mechanical FES, the pathologic reaction changes from a mechanical obstruction to a biochemical process. However, no study to date has examined the relationship between these two reactions during the development of FES. In the present study, we experimentally induced FES by injecting triolein $(700 \mathrm{mg} / \mathrm{kg})$ into 25 rabbits and observed the disease progression. Eight animals died immediately after the injection, while another six developed dyspnea and confusion and died 7-60 hours after the injection. The other 11 recovered from tachypnea and survived to the end of the experiment.

The rabbits that died immediately after the injection had pale lungs and right ventricular hypertrophy. Histological examinations revealed large fat vacuoles within large pulmonary vessels, but normal lung parenchyma. These findings suggest mechanical obstruction of the large pulmonary artery by the injected triolein. Further, blockage of the blood flow through the lung caused right-side heart failure and sudden death [10]. These findings suggest that the rabbits died of fulminant-acute FES due to the triolein injection.

The rabbits that died 7-60 hours after the injection of triolein showed diffuse hemorrhage and edematous fluid in the alveoli and hemorrhagic exudate in the pleural cavity, similar to the typical findings of FFA-induced FES [10].

The rabbits that survived until the end of the experiment recovered from tachypnea. Histological examination of the lungs showed mixed features of normal lung tissue and hemorrhagic lesions. Many macrophages were visible under the microscope as phagocytosing red blood cells and absorbing extravasated blood filling the alveoli. 
These findings suggest that these rabbits were recovering from lung hemorrhage, rather than from obstruction.

The result of blood tests over time in the surviving rabbits showed that the FFA and FFA/albumin levels increased by up to $40 \%$, whereas TG and lipase levels slightly decreased below the initial level during the first 12 hours. This suggests that the fat emboli were hydrolyzed to FFA more rapidly than expected. After 12 hours, the FFA and FFA/albumin levels rapidly decreased, whereas the TG level began to increase markedly. After 48 hours, the reduced FFA and FFA/albumin levels began to increase slightly and returned to the pre-injection level (Fig. 7).

Altogether, eight of 25 rabbits died immediately of mechanical obstruction; six died of FES by toxic effects due to markedly increased FFA levels induced by the injected triolein; and 11 recovered from FES associated with a decreased FFA level, which increased up to $40 \%$ in the early phase of the experiment. These clinical, histological, and biochemical results are consistent with the hypothesis of this study that fat emboli, which mechanically obstruct the alveolar capillaries, are hydrolyzed to FFAs, the toxic effects of which cause sub-acute FES.

These results align well with the clinical course of FES. The rabbits that died within 1 hour functioned as models of quickly progressing fulminant-acute FES. The rabbits that died 7-60 hours after the injection were models of sub-acute FES, which usually occurs 12-48 hours after injury in the clinical setting. We stopped this study at 72 hours because the incidence of FES after this time period is very rare and none of the surviving rabbits had clinical symptoms.

In the hydrolysis process, alveolar cells can produce lipase, which is believed to hydrolyze fat emboli to FFAs if the lung alveolar capillaries are obstructed $[3,23]$. Thus, the initial decrease in lipase levels that occurred for up to 24 hours was probably due to a consumption-related decrease in existing lipase in the bloodstream during hydrolysis of the injected triolein. A possible explanation for the increasing lipase levels after 24 hours may be that lipase was produced by the lung and released into the bloodstream by reperfusion.

Another point to note in the present study is that the FFA concentrations in the rabbits that died at 7-60 hours after injection showed rapid and tremendous increases. This finding suggests that the onset of sub-acute FES was determined by how many of the fat emboli were transformed to FFAs and how rapidly this transformation took place.

In the present study, the increase in FFA by hydrolysis of fat emboli was among the important causes of tissue damage in FES. Further studies are needed to identify ways of reducing the blood FFA levels, tissue damage, and mortality rate.

In conclusion, the two hypotheses of FES, mechanical obstruction and biochemical processes, can be generally accepted. The present study is the first to describe these two hypotheses as being interrelated, rather than independent. Fat emboli initially act as mechanical obstructors, but are gradually hydrolyzed to form toxic FFAs, inducing sub-acute FES.

The present study revealed the pathophysiology of FES in more detail. These results are expected to help predict the occurrence of FES and aid in the development of more effective treatments for it.

\section{NOTES}

\section{Conflict of interest}

No potential conflict of interest relevant to this article was reported.

\section{Ethical approval}

The study was approved by the Institutional Animal Care and Use Committee in Good Moonhwa Hospital (IACUC No. 2013-02) and followed the principles of scientific research ethics.

\section{ORCID}

Hong Il Kim

Seok Kyung In

Hyung Suk Yi

Hyo Young Kim

Yoon Soo Kim

\section{REFERENCES}

1. Aust L, Devlin B, Foster SJ, et al. Yield of human adipose-derived adult stem cells from liposuction aspirates. Cytotherapy 2004;6:7-14.

2. Lee WS, Park SH, Kang SG, et al. Emergency room visits for severe complications after cosmetic surgery. Arch Aesthetic Plast Surg 2019; 25:108-14.

3. Mellor A, Soni N. Fat embolism. Anaesthesia 2001;56:145-54.

4. Zenker FA. Fett-Embolie der Lungencapillaren. In: Zenker FA, editor. Beiträge zur normalen und pathologischen Anatomie der Lunge. Dresden: J. Braunsdorf; 1862. p. 31.

5. Filomeno LT, Carelli CR, Figueiredo da Silva NC, et al. Fat embolism: a review for current orthopaedics practice. Acta Orhtop Bras 2005;13: 196-208.

6. Mastrangelo AM, Jeitner TM, Eaton JW. Oleic acid increases cell surface expression and activity of CD11b on human neutrophils. J Immunol 1998;161:4268-75.

7. Saigal R, Mittal M, Kansal A, et al. Fat embolism syndrome. J Assoc Physicians India 2008;56:245-9.

8. Gauss H. The pathology of fat embolism. Arch Surg 1924;9:593-605.

9. Lehman EP, Moore RM. Fat embolism: including experimental production without trauma. Arch Surg 1927;14:621-62.

10. Peltier LF. Fat embolism. III. The toxic properties of neutral fat and free fatty acids. Surgery 1956;40:665-70.

11. Laub DR Jr, Laub DR. Fat embolism syndrome after liposuction: a case report and review of the literature. Ann Plast Surg 1990;25:48-52. 
12. Pell AC, Hughes D, Keating J, et al. Brief report: fulminating fat embolism syndrome caused by paradoxical embolism through a patent foramen ovale. N Engl J Med 1993;329:926-9.

13. Scully RE. Fat embolism in Korean battle casualties; its incidence, clinical significance, and pathologic aspects. Am J Pathol 1956;32:379-403.

14. Hwang SM, Lee JS, Kim HI, et al. Transposition of intravascular lipid in experimentally induced fat embolism: a preliminary study. Arch Plast Surg 2014;41:325-9.

15. Burnstein RM, Newell JP, Jones JG. Sequential changes in gas exchange following traumatic fat embolism. Anaesthesia 1998;53:373-8.

16. Capan LM, Miller SM, Patel KP. Fat embolism. Anesthesiol Clin North Am 1993;11:25-54.

17. Robinson CM. Current concepts of respiratory insufficiency syndromes after fracture. J Bone Joint Surg Br 2001;83:781-91.

18. Scroggins C, Barson PK. Fat embolism syndrome in a case of abdomi- nal lipectomy with liposuction. Md Med J 1999;48:116-8.

19. Wang HD, Zheng JH, Deng CL, et al. Fat embolism syndromes following liposuction. Aesthetic Plast Surg 2008;32:731-6.

20. Gravante G, Araco A, Sorge R, et al. Pulmonary embolism after combined abdominoplasty and flank liposuction: a correlation with the amount of fat removed. Ann Plast Surg 2008;60:604-8.

21. Ross RM, Johnson GW. Fat embolism after liposuction. Chest 1988;93: 1294-5.

22. Costa AN, Mendes DM, Toufen C, et al. Adult respiratory distress syndrome due to fat embolism in the postoperative period following liposuction and fat grafting. J Bras Pneumol 2008;34:622-5.

23. Gossling HR, Pellegrini VD Jr. Fat embolism syndrome: a review of the pathophysiology and physiological basis of treatment. Clin Orthop Relat Res 1982;(165):68-82. 